\title{
The Semi-Generic Group Model and Applications to Pairing-Based Cryptography ${ }^{\star}$
}

\author{
Tibor Jager ${ }^{1}$ and Andy Rupp ${ }^{2}$ \\ 1 Horst Görtz Institute for IT Security, Ruhr-University Bochum, Germany \\ tibor.jagerarub. de \\ ${ }^{2}$ University of Trier, Germany \\ andy.rupp@rub.de
}

\begin{abstract}
In pairing-based cryptography the Generic Group Model (GGM) is used frequently to provide evidence towards newly introduced hardness assumptions. Unfortunately, the GGM does not reflect many known properties of bilinear group settings and thus hardness results in this model are of limited significance. This paper proposes a novel computational model for pairing-based cryptography, called the Semi-Generic Group Model (SGGM), that is closer to the standard model and allows to make more meaningful security guarantees. In fact, the best algorithms currently known for solving pairing-based problems are semi-generic in nature. We demonstrate the usefulness of our new model by applying it to study several important assumptions (BDDH, Co-DH). Furthermore, we develop master theorems facilitating an easy analysis of other (future) assumptions. These master theorems imply that (unless there are better algorithms than the semigeneric ones) great parts of the zoo of novel assumptions over bilinear groups are reducible to just two (more or less) standard assumptions over finite fields. Finally, we examine the appropriateness of the SGGM as a tool for analyzing the security of practical cryptosystems without random oracles by applying it to the BLS signature scheme.
\end{abstract}

Keywords: Restricted models of computation, generic groups, semi-generic group model, cryptographic assumptions, master theorems, provable security, pairingbased cryptography.

\section{Introduction}

Assuming that certain computational problems, mostly from algebra, number theory, and coding theory, are intractable builds the foundation of public-key cryptography. However, proving the validity of these assumptions in the standard model of computation seems to be impossible with currently available techniques.

Why do we believe in such hardness assumptions, though they are not provable in general? For classic number-theoretic problems, such as integer factorization (IF) or the discrete logarithm (DL) problem, this is certainly due to the absence of efficient

\footnotetext{
* This is an extended abstract, see [20] for the full version. This research has been supported by the European Community (FP7/2007-2013) under grant agreement number ICT-2007-216646 - European Network of Excellence in Cryptology II (ECRYPT II).
} 
algorithms in spite of intensive long-term research by many brilliant people. However, besides such well-known assumptions, there frequently appear new assumptions building the basis for novel cryptosystems with original properties. What can be done to provide evidence for these assumptions apart from trying to find efficient algorithms over decades? Clearly, we should try to underpin the belief in novel assumptions by searching for reductions to a more mature assumption; but unfortunately finding such a reduction often fails.

An important approach to (nevertheless) gain immediate evidence towards hardness assumptions is to prove them with respect to a restricted but still meaningful class of algorithms. This is the motivation behind the invention of black-box models for algebraic structures like groups, fields, and rings, where algorithms are limited to perform only operations "commonly" available over these structures. Probably, the most famous of these models is the generic group model (GGM) introduced by Shoup in his seminal paper [34] from 1997, and refined by Maurer in [28]. In this model one considers algorithms - so-called generic group algorithms - that, given a group $\mathbb{G}$ as black-box, may only perform a set of basic operations on the elements of $\mathbb{G}$ such as applying the group law, inversion of group elements and equality testing. Since the group is treated as a black-box, the algorithms cannot exploit any special properties of a concrete group representation. As a consequence, such algorithms are generic in the sense that they can be applied to any concrete instantiation of a group (e.g., $\mathbb{Z}_{p}^{*}$ or $E\left(\mathbb{F}_{p}\right)$ ) in order so solve a problem. Natural examples of this class of algorithms are the Pohlig-Hellman [30] and Pollard's Rho [31] algorithm for computing discrete logarithms.

It should be noted that one has to take care when interpreting results in the GGM like intractability results as evidence in practice, since this model abstracts away from potentially many properties an algorithm might be able to exploit in the real world [15]. On the one hand, there exist cryptographic groups (such as certain elliptic curve groups) for which not many properties beyond the axioms of an algebraic group are known. Hence, modeling such groups as generic can be seen as a reasonable abstraction. On the other hand, there are groups, also used in cryptography, featuring many further properties, which clearly makes the generic model an inappropriate reflection for them. A prime example are multiplicative groups of finite fields or rings. These structures offer many well-understood properties beyond the group axioms, such as additional efficient algebraic operations (e.g., addition in the field or ring), and other properties of the group representation (e.g., the notion of prime integers and irreducible polynomials), that are simply ignored by the generic group model, but give rise to more efficient algorithms for certain problems (e.g., index calculus algorithms for computing discrete logarithms).

But should a minimal requirement on such an idealized model of computation not be that at least all currently known algorithms are captured? There exist some first approaches in the cryptographic literature to tackle this issue: The pseudo-free group model proposed by Hohenberger [19] and Rivest [32] does not treat a group as a blackbox. Unfortunately, the definition of pseudo-freeness is very restrictive in the sense that a number of important groups (like all known-order groups) are immediately excluded and important problems, such as Diffie-Hellman-type problems, seem not to be covered. Other approaches due to Leander and Rupp [27] and Aggarwal and Maurer [1] take into account that the RSA group $\mathbb{Z}_{n}^{*}$ is embedded in the ring $\mathbb{Z}_{n}$. They use 
a generic ring model, where algorithms may perform both multiplication and addition operations on $\mathbb{Z}_{n}$ to show that breaking RSA is equivalent to factoring. Unfortunately, recent work [21] shows that even computing the Jacobi symbol is equivalent to factoring in this model. So this approach has not led to a satisfying abstraction of $\mathbb{Z}_{n}^{*}$ yet.

Over the last decade a considerable number of innovative cryptosystems, such as identity-based encryption [7] or short digital signatures with strong security [910], have been proposed over bilinear groups. A bilinear group setting consists of groups $\mathbb{G}_{1}, \mathbb{G}_{2}$, and $\mathbb{G}_{3}$, with a bilinear map $e: \mathbb{G}_{1} \times \mathbb{G}_{2} \rightarrow \mathbb{G}_{3}$, called a pairing. Along with these cryptosystems also many new assumptions have been introduced, e.g., Bilinear Diffie-Hellman (BDH) [23 24], $q$-Strong Diffie-Hellman [4 14 22], Decision Linear Diffie-Hellman (DLIN) [6], Co-Diffie-Hellman (Co-DH) [9]8], and many more. Unfortunately, for virtually all of them no reduction to a well-analyzed assumption like DL is known. In fact, finding such reductions seems to be a difficult task, since the algebraic settings underlying classic problems (e.g., a single cyclic group for DL) significantly differ from bilinear settings. Hence, given an instance of a classic problem, it appears to be hard to transform this instance to one of the bilinear problem in order to leverage an algorithm for the latter.

Consequently, the only way to provide some immediate evidence for such novel assumptions consists in proofs in restricted models of computation. So far, the only such model for bilinear settings is a straightforward extension of the generic group model, where all three groups $\mathbb{G}_{1}, \mathbb{G}_{2}$, and $\mathbb{G}_{3}$ are modeled as generic groups [33|11|25]. In all known instances of bilinear settings the groups $\mathbb{G}_{1}$ and $\mathbb{G}_{2}$ are elliptic curve groups, thus modeling these groups as generic may be considered as a reasonable abstraction. However, in contrast to that, the group $\mathbb{G}_{3}$ is usually a subgroup of the multiplicative group of a finite field. So there definitely exist non-generic algorithms for cryptographic problems like BDH, Co-DH, etc. featuring a running time which is at most sub-exponential: these sub-exponential algorithms map the inputs over $\mathbb{G}_{1}$ and $\mathbb{G}_{2}$ (given as part of a problem instance) to $\mathbb{G}_{3}$ using the bilinear mapping (MOV reduction [29]) and determine the discrete logarithms of these elements over $\mathbb{G}_{3}$ using index calculus. Knowledge of these discrete logarithms allows to compute the solution to the problem instance using a few exponentiations. Note that there might be even more efficient algorithms especially for potentially easier problems like decisional or gap problems. Hence, modeling bilinear settings in this way is clearly inappropriate.

Our Contribution. We propose the Semi-Generic Group Model (SGGM) which leverages this observation as follows: The elliptic curve groups $\mathbb{G}_{1}$ and $\mathbb{G}_{2}$ are modeled as generic groups, while $\mathbb{G}_{3}$ is given in the standard model, i.e., algorithms may perform any computation over $\mathbb{G}_{3}$ that is possible in the subgroup of a finite field. The SGGM is thus closer to the standard model than the GGM and can provide stronger evidence towards hardness assumptions in pairing-based cryptography. In fact, to the best of our knowledge all algorithms currently known for solving pairing-based problems are semi-generic in nature. In particular, the sub-exponential algorithms applying a MOV reduction described above are covered by the SGGM.

We analyzed some of the most important computational and decisional assumptions of pairing-based cryptography in our new model. In this extended abstract we restrict to consider Co-DH and decisional BDH. The full version of the paper [20] covers 
additional problems, including $q$-strong DH and DLIN. We are able to reduce the considered assumptions (with respect to semi-generic algorithms) to fairly standard assumptions over finite fields like Square DH and a slight variation of DL. That means, the bilinear assumptions are at least as hard as certain more standard assumption over $\mathbb{G}_{3}$ provided that there are no non-semi-generic algorithms. Furthermore, we developed master theorems ensuring the hardness of broad classes of computational and decisional problems in the SGGM. Studying such generalizations is not only important in order to structure and facilitate the analysis of the rapidly growing set of cryptographic assumptions as motivated in [3], but improves our understanding of the properties which need to be satisfied by a problem to be intractable. Results like [12 3311] are in this vein. Boyen [11] (see also [5]) developed master theorems for the hardness of some general classes of decisional problems in the generic group model for bilinear settings. Rupp et al. [33] provide hardness conditions for even broader classes of computational problems and algebraic settings, but still in the GGM. Bresson et al. [12] study a general class of decisional assumptions over a single group in the standard model and show that this class can be reduced to DDH (under certain restrictions). In the scope of the proof of our master theorem for decisional problems we enhance Bresson et al.'s results for the standard model and apply them to the SGGM.

The security of public-key cryptosystems, especially of practical cryptosystems, can often only be proven in an idealized model, such as the random oracle model (ROM) [2]. An issue with the ROM is that it idealizes a hash function in a way such that it has all properties of a "perfect" hash function (collision resistance, (second) preimage resistance, random output, ...) at the same time. When the cryptosystem (and thus the random oracle) is implemented in practice, one has to choose an adequate hash function instantiating the random oracle. An important question is whether providing all properties of the random oracle at the same time is really necessary to provide security.

We examine the useability of the SGGM as a tool complementing the ROM. We are able to prove the security of the Boneh-Lynn-Shacham (BLS) short signature scheme [9 10] against semi-generic adversaries without random oracles, however, requiring non-standard properties for the employed hash function. It is left as an interesting open problem to study whether these requirements can actually be satisfied by a reasonably efficient practical hash function.

\section{The Semi-Generic Group Model}

Let $\mathbb{G}_{1}, \mathbb{G}_{2}$, and $\mathbb{G}_{3}$ be groups of prime order $p$ and $g_{1} \in \mathbb{G}_{1}, g_{2} \in \mathbb{G}_{2}$ be corresponding generators. For the sake of simplicity of the subsequent formalizations we use multiplicative notation for all groups.

Definition 1. A pairing is a map e : $\mathbb{G}_{1} \times \mathbb{G}_{2} \rightarrow \mathbb{G}_{3}$ with the following properties:

1. Bilinearity: $\forall(a, b) \in \mathbb{G}_{1} \times \mathbb{G}_{2}$ and $x_{1}, x_{2} \in \mathbb{Z}_{p}$ holds that $e\left(a^{x_{1}}, b^{x_{2}}\right)=e(a, b)^{x_{1} x_{2}}$.

2. Non-degeneracy: $g_{3}:=e\left(g_{1}, g_{2}\right)$ is a generator of $\mathbb{G}_{3}$, i.e., $g_{3} \neq 1$.

3. $e$ is efficiently computable. 
Following [17], we distinguish three different types of bilinear group settings:

- Type 1: $\mathbb{G}_{1}=\mathbb{G}_{2}$. We will call this the setting with symmetric bilinear map.

- Type 2: $\mathbb{G}_{1} \neq \mathbb{G}_{2}$, there is an efficiently computable isomorphism $\psi: \mathbb{G}_{1} \rightarrow \mathbb{G}_{2}$.

- Type 3: $\mathbb{G}_{1} \neq \mathbb{G}_{2}$, there is no efficiently computable isomorphism $\psi: \mathbb{G}_{1} \rightarrow \mathbb{G}_{2}$.

Formal Definition of THE SGGM. We base our formal description of the SGGM for bilinear settings on the generic group model introduced by Maurer [28], though our proofs can be adapted to Shoup's GGM [34] as well. The main difference between Maurer's and Shoup's formalization is that in the first model group elements are encoded deterministically whereas in the second model encodings are random.

An algorithm $\mathcal{A}$ in the SGGM interacts with a semi-generic group oracle $\mathcal{O}$, which computes the group operations and evaluates the pairing and isomorphism on behalf of $\mathcal{A}$. $\mathcal{O}$ receives as input two vectors of group elements (the problem instance)

$$
I_{1}=\left(a_{1,1}, \ldots, a_{1, k_{1}}\right) \in \mathbb{G}_{1}^{k_{1}} \quad \text { and } \quad I_{2}=\left(a_{2,1}, \ldots, a_{2, k_{2}}\right) \in \mathbb{G}_{2}^{k_{2}} .
$$

It maintains two lists $\mathcal{E}_{1} \subseteq \mathbb{G}_{1}$ and $\mathcal{E}_{2} \subseteq \mathbb{G}_{2}$, with $\mathcal{E}_{i, j}$ denoting the $j$-th entry of list $\mathcal{E}_{i}$, which are initialized such that $\mathcal{E}_{i, j}:=a_{i, j}$ for all possible $(i, j)$. We denote with $[a]_{i}$ the smallest index $j$ (also called encoding) such that $\mathcal{E}_{i, j}=a$. Index $[a]_{i}$ is undefined, if $a \notin \mathcal{E}_{i}$. We may always assume that semi-generic algorithms only provide defined indices as input to the oracle. During initialization of the lists $\mathcal{E}_{1}$ and $\mathcal{E}_{2}$, the corresponding indices pointing to the contained elements are sent to the algorithm.

The oracle implements the following public procedures, which may be called by $\mathcal{A}$ :

- GroupOp $\left([a]_{i},[b]_{i}, i\right)$ : This procedure takes as input two indices $[a]_{i},[b]_{i}$ and a list index $i$. It determines the group elements $a, b \in \mathbb{G}_{i}$ by list lookup, computes $c=$ $a \cdot b \in \mathbb{G}_{i}$, appends $c$ to $\mathcal{E}_{i}$, and returns $[c]_{i}$.

- BilinearMap $\left([a]_{1},[b]_{2}\right)$ : This procedure takes as input two indices $[a]_{1},[b]_{2}$. It determines the corresponding group elements $a \in \mathbb{G}_{1}, b \in \mathbb{G}_{2}$ by list lookup and returns $e(a, b)$ in the standard representation of $\mathbb{G}_{3}$ (i.e., as finite field element).

When considering Type 2 settings the algorithm may also query to apply the isomorphism $\psi$ to an element of $\mathbb{G}_{1}$ :

- Isomorphism $\left([a]_{1}\right)$ : This procedure takes as input an index $[a]_{1}$, determines the element $a \in \mathbb{G}_{1}$, computes $b=\psi(a)$, appends $b$ to $\mathcal{E}_{2}$ and returns $[b]_{2}$.

Note that a random group element can be efficiently sampled by a semi-generic algorithm by using GroupOp(.) to raise the generator (which is always part of a problem instance) to some $r \stackrel{\$}{\leftarrow} \mathbb{Z}_{p}$.

\subsection{Essential Ingredients for Proofs in the SGGM}

This section describes a few general observations that will turn out to be the essential ingredients for proofs in the semi-generic model.

Observation 1: Components inside oracle are exchangeable. Semi-generic algorithms due to its nature are "blind" with respect to the internal details of the groups 
$\mathbb{G}_{1}$ and $\mathbb{G}_{2}$ as well as the pairing $e$ and the isomorphism $\psi$. These components are hidden within a black-box. Hence, we can plug-in "something else" for these components as long as these replacements behave like cyclic groups with a bilinear map and an isomorphism. We will utilize this observation in a novel way to map inputs given over $\mathbb{G}_{3}$ back to $\mathbb{G}_{1}$ and $\mathbb{G}_{2}$ by setting $\mathbb{G}_{1}:=\mathbb{G}_{2}:=\mathbb{G}_{3}$ internally and simulating a virtual bilinear map $e: \mathbb{G}_{3} \times \mathbb{G}_{3} \rightarrow \mathbb{G}_{3}$ and isomorphism $\psi: \mathbb{G}_{3} \rightarrow \mathbb{G}_{3}$.

Observation 2: Computed elements over $\mathbb{G}_{1}$ and $\mathbb{G}_{2}$ are linear polynomials in initial inputs. Let $I_{1} \in \mathbb{G}_{1}^{m}$ and $I_{2} \in \mathbb{G}_{2}^{n}$ be inputs given to the semi-generic oracle (as part of a problem instance). We have $I_{2}=I_{1}$ in the case of a Type 1 setting. In the following, we always assume that at least the generators $g_{1}$ and $g_{2}$ are given (as the first components of these input tuples). So we can write $I_{1}=\left(g_{1}, g_{1}^{x_{2}}, \ldots, g_{1}^{x_{m}}\right)$ and $I_{2}=\left(g_{2}, g_{2}^{y_{2}}, \ldots, g_{2}^{y_{n}}\right)$ for some unknown $x_{j}, y_{k} \in \mathbb{Z}_{p}$ (no assumptions about their distribution are made here) and $\psi\left(g_{1}\right)=g_{2}$ in the case of a Type 2 setting. Then we define the tuple $I_{1}^{\prime}:=I_{1}$ and the tuple $I_{2}^{\prime}:=I_{2}$ in the case of a Type 1 and Type 3 setting or $I_{2}^{\prime}:=\left(g_{2}, g_{2}^{x_{2}}, \ldots, g_{2}^{x_{m}}, g_{2}^{y_{2}}, \ldots, g_{2}^{y_{n}}\right)$ for a Type 2 setting. These tuples are called the initial inputs to semi-generic algorithms. Using this notation, we can describe the following observation: Over $\mathbb{G}_{1}$ and $\mathbb{G}_{2}$ a semi-generic algorithm can only perform the group law on the initial inputs. Thus, any element $a \in \mathbb{G}_{i}(i \in\{1,2\})$ computed by a semi-generic algorithm is a product of the elements in $I_{i}^{\prime}$. Hence we can represent such an element as $a=g_{i}^{P\left(x_{2}, \ldots, x_{m}, y_{2}, \ldots, y_{n}\right)}$ for some linear multivariate polynomial $P=\alpha_{1}+\sum_{j=2}^{m} \alpha_{j} X_{j}+\sum_{j=2}^{n} \beta_{j} Y_{j}$, where the $\beta_{j}$ are zero in the case $i=1$ or if we consider a Type 1 setting. It is important to observe that all coefficients of this polynomial are known to the oracle.

Observation 3: Pairing is simulatable knowing images of initial inputs. Let $a \in \mathbb{G}_{1}$ and $b \in \mathbb{G}_{2}$ be two elements computed by a semi-generic algorithm. Then by using the above observation and setting $x_{1}:=1$ it is easy to see that

$$
\begin{aligned}
e(a, b) & =e\left(g_{1}^{\sum_{i=1}^{m} \alpha_{i} x_{i}}, g_{2}^{\sum_{j=1}^{m} \alpha_{j}^{\prime} x_{j}+\sum_{k=2}^{n} \beta_{k}^{\prime} y_{k}}\right) \\
& =\prod_{i=1}^{m} \prod_{j=1}^{m} e\left(g_{1}^{x_{i}}, g_{2}^{x_{j}}\right)^{\alpha_{i} \alpha_{j}^{\prime}} \cdot \prod_{i=1}^{m} \prod_{k=2}^{n} e\left(g_{1}^{x_{i}}, g_{2}^{y_{k}}\right)^{\alpha_{i} \beta_{k}^{\prime}}
\end{aligned}
$$

From this equation it follows that by knowing the images of the initial inputs under the pairing, one can compute the output of e on arbitrary inputs provided by a semi-generic algorithm without actually evaluating the pairing explicitly. In other words, an oracle equipped with a table containing $e(a, b)$ for all combinations of $a$ in $I_{1}^{\prime}$ and $b$ in $I_{2}^{\prime}$ would be able to handle all BilinearMap queries.

\section{Analysis of Selected Problems in the Semi-Generic Model}

In this section we exemplarily analyze the hardness of the computational Co-DH and the decisional BDH problem. Certainly, the list of problems we are considering here is by no means complete. Our main purpose is to give concrete analyses of some important problems of bilinear cryptography, thereby illustrating the basic ideas and techniques underlying proofs in this model, before dealing with the more intricate case of general classes of problems in Section 4 


\subsection{Reducing 2-DL to Co-DH}

The Co-DH problem has been used in [9]8] for the construction of short and aggregate signatures over bilinear groups. Over a Type 2 setting it can be defined as follows: Given $\left(g_{1}, g_{1}^{x_{0}}, g_{2}, g_{2}^{x_{1}}, g_{3}\right)$, where $\left(x_{0}, x_{1}\right) \stackrel{\$}{\leftarrow} \mathbb{Z}_{p}^{2}$ are secret random choices, output $g_{2}^{x_{0} x_{1}}$.

It is easy to see that in order to prove something about the hardness of Co-DH, we definitely need to make the assumption that the discrete logarithm problem over $\mathbb{G}_{3}$ is intractable. But is this enough? Our answer is "not quite": We are going to relate the hardness of $\mathrm{Co}-\mathrm{DH}$ to the 2 -DL problem over $\mathbb{G}_{3}$, a slightly easier variant of DL. The $q$-DL problem can be defined as follows: Given $\left(g_{3}, g_{3}^{x^{1}}, \ldots, g_{3}^{x^{q}}\right)$, where $x \stackrel{\$}{\leftarrow} \mathbb{Z}_{p}$ is a secret random value, output $x$. The additional input $g_{3}^{x^{2}}$ (in comparison to standard DL) is needed in order to be able to simulate the pairing when running the $\mathrm{Co}-\mathrm{DH}$ algorithm.

Theorem 1. Suppose there exists a semi-generic group algorithm $\mathcal{A}$ solving $\mathrm{Co}-\mathrm{DH}$ over a Type 2 bilinear group setting in time $t$ with success probability $\epsilon$. Then there exists an algorithm $\mathcal{B}$ solving the 2 -DL problem over $\mathbb{G}_{3}$ in time $t^{\prime} \approx t$ with success probability $\epsilon^{\prime} \geq \frac{1}{2} \epsilon$.

Proof. Given an instance of the 2-DL problem, $\mathcal{B}$ sets up an instance of the Co-DH problem in the semi-generic model in a way that it can leverage a solution to $\mathrm{Co}-\mathrm{DH}$ computed by $\mathcal{A}$ to solve the 2 -DL instance. In particular, $\mathcal{B}$ will play the role of the semi-generic oracle. We exploit Observation 1 from Section 2.1 to setup such an useful instance: Since $\mathcal{A}$ is "blind" with respect to the internal details of $\mathbb{G}_{1}, \mathbb{G}_{2}, e$, and $\psi$, we set $\mathbb{G}_{1}:=\mathbb{G}_{2}:=\mathbb{G}_{3}$ and try to simulate a virtual bilinear map $e: \mathbb{G}_{3} \times \mathbb{G}_{3} \rightarrow \mathbb{G}_{3}$.

We are now ready to describe our reduction algorithm $\mathcal{B}$. $\mathcal{B}$ takes as input an instance $a_{0}:=g_{3}, a_{1}:=g_{3}^{x}, a_{3}:=g_{3}^{x^{2}}$ of the 2-DL problem over $\mathbb{G}_{3}$. Then it chooses $i^{*} \stackrel{\$}{\leftarrow}$ $\{0,1\}, x_{1-i^{*}} \stackrel{\$}{\leftarrow} \mathbb{Z}_{p}$ and sets $a_{2}:=g_{3}^{x_{1-i^{*}}}$. The wanted discrete logarithm $x$ is now embedded as the implicit secret choice $x_{i^{*}}$ in an instance of the Co-DH problem. More precisely, $\mathcal{B}$ sets up a problem instance and simulates the oracle $\mathcal{O}$ as follows:

- The lists $\mathcal{E}_{1}$ and $\mathcal{E}_{2}$ are initialized with $g_{3}, g_{3}^{x_{0}}$ and $g_{3}, g_{3}^{x_{1}}$, respectively, where $g_{3}^{x_{i} \text { * }}$ is set to be $a_{1}$. The indices $\left[g_{3}\right]_{1},\left[g_{3}^{x_{0}}\right]_{1},\left[g_{3}\right]_{2},\left[g_{3}^{x_{1}}\right]_{2}$, and $g_{3}$ are sent out to $\mathcal{A}$.

- Group0p can be simulated since $\mathcal{B}$ knows how to perform the group law over $\mathbb{G}_{3}$.

- Isomorphism $\left([a]_{1}\right)$ can be simulated by looking up $a$ in $\mathcal{E}_{1}$, appending it to $\mathcal{E}_{2}$, and then determining the index $[a]_{2}$.

- Using Observation 3 from Section 2.1, we can easily see that BilinearMap can be simulated: Let $[b]_{1},[c]_{2}$ be the two indices given as input to the procedure by $\mathcal{A}$. Then we can write

$$
e(b, c)=e\left(g_{3}^{\sum_{j=-1}^{0} z_{j} x_{j}}, g_{3}^{\sum_{k=-1}^{1} z_{k}^{\prime} x_{k}}\right)=\prod_{j=-1}^{0} \prod_{k=-1}^{1}\left(g_{3}^{x_{j} x_{k}}\right)^{z_{j} z_{k}^{\prime}}
$$

where $x_{-1}:=1$ and $z_{j}$ and $z_{k}^{\prime}$ are known to $\mathcal{B}$. Since $\mathcal{B}$ is given $a_{0}, \ldots, a_{3}$ and knows $i^{*}, x_{1-i^{*}}$, it can compute the required elements $g_{3}, g_{3}^{x_{0}}, g_{3}^{x_{1}}, g_{3}^{x_{0}^{2}}, g_{3}^{x_{0} x_{1}}$ to simulate the pairing: $g_{3}=a_{0}, g_{3}^{x_{i^{*}}}=a_{1}, g_{3}^{x_{1-i^{*}}}=a_{2}, g_{3}^{x_{0}^{2}}=a_{3}$ if $i^{*}=0$ and $g_{3}^{x_{0}^{2}}=a_{2}^{x_{0}}$ else, $g_{3}^{x_{0} x_{1}}=a_{1}^{x_{1-i^{*}}}$. 
Given some instance of Co-DH, algorithm $\mathcal{A}$ eventually outputs some valid index $[c]_{2}$. The corresponding element $c \in \mathbb{G}_{2}$ can be written as $c=g_{2}^{P\left(x_{0}, x_{1}\right)}$ for some known polynomial $P=z_{0}+z_{1} X_{0}+z_{2} X_{1} \in \mathbb{Z}_{p}\left[X_{0}, X_{1}\right]$ (Observation 2, Section 2.1). So alternatively we can say that $\mathcal{A}$ wins if $\left(P-X_{0} X_{1}\right)\left(x_{0}, x_{1}\right) \equiv 0 \bmod p$. This success event can be split up into the following disjoint events:

- Event $\mathcal{S}_{1}$ : The univariate polynomial $\left(P-X_{0} X_{1}\right)\left(x_{0}\right)$, i.e., the polynomial $P$ $X_{0} X_{1}$ where we only evaluate the variable $X_{0}$ with $x_{0}$, is zero modulo $p$. Let the probability of this event be denoted by $\alpha_{1}$.

- Event $\mathcal{S}_{2}$ : The univariate polynomial $\left(P-X_{0} X_{1}\right)\left(x_{0}\right)$ is not zero modulo $p$ but $\left(P-X_{0} X_{1}\right)\left(x_{0}, x_{1}\right)$ is. Let the probability of this event be denoted by $\alpha_{2}$.

Clearly, we have $\epsilon=\alpha_{1}+\alpha_{2}$.

Let us consider the events $\mathcal{S}_{1}$ and $\mathcal{S}_{2}$ when $\mathcal{B}$ runs $\mathcal{A}$ for certain choices of $i^{*}$. Note that $\mathcal{B}$ knows the coefficients of $P$ since it responded to $\mathcal{A}$ 's queries. With probability $\frac{1}{2} \alpha_{1}$, we have $i^{*}=0$ and $\mathcal{S}_{1}$. This means $z_{0}+z_{1} x+z_{2} X_{1}-x X_{1} \equiv 0$. But in this case $z_{2}$ needs to be equal to $x$. So $\mathcal{B}$ wins by simply returning the known coefficient $z_{2}$. Furthermore, with probability $\frac{1}{2} \alpha_{2}$, we have $i^{*}=1$ and $\mathcal{S}_{2}$. Hence, the wanted DL is the root of the uni-variate non-zero polynomial $z_{0}+z_{1} x_{0}+z_{2} X_{1}-x_{0} X_{1}$ known to $\mathcal{B}$. It can thus be determined as $x \equiv\left(z_{0}+z_{1} x_{0}\right)\left(x_{0}-z_{2}\right)^{-1} \bmod p$. It is easy to verify that the inverse $\left(x_{0}-z_{2}\right)^{-1}$ always exists.

To summarize, if $i^{*}$ happens to be zero, $\mathcal{B}$ outputs $z_{2}$, otherwise it outputs $\left(z_{0}+\right.$ $\left.z_{1} x_{0}\right)\left(x_{0}-z_{2}\right)^{-1}$. In this way, its success probability is at least $\frac{1}{2} \alpha_{1}+\frac{1}{2} \alpha_{2}=\frac{1}{2} \epsilon$.

\subsection{Reducing SqDDH to BDDH}

The bilinear decisional Diffie-Hellman problem (BDDH) is certainly among the most well-known problems over bilinear groups. It has originally been introduced in a seminal paper by Joux [23] and, e.g., further been used by Boneh and Franklin [7] to construct an identity based encryption scheme. Let us consider BDDH over a Type 1 setting where it can be defined as follows: Given $\left(g_{1}, g_{1}^{x_{1}}, g_{1}^{x_{2}}, g_{1}^{x_{3}}, g_{3}^{r_{b}}\right)$, where $\left(x_{1}, x_{2}, x_{3}\right) \stackrel{\$}{\leftarrow}$ $\mathbb{Z}_{p}^{3}, b \stackrel{\&}{\leftarrow}\{0,1\}, r_{1}=x_{1} x_{2} x_{3}$, and $r_{0} \stackrel{\&}{\leftarrow} \mathbb{Z}_{p}$ are secret choices, output $b$.

We relate the hardness of BDDH with respect to semi-generic algorithms to the hardness of the well-known decisional Diffie-Hellman (DDH) problem and the square decisional Diffie-Hellman $(\mathrm{SqDDH})$ problem over $\mathbb{G}_{3}$. $\mathrm{SqDDH}$ is a potentially easier variant of DDH: Given $\left(g_{3}, g_{3}^{x}, g_{3}^{r_{b}}\right)$, where $x \stackrel{\$}{\leftarrow} \mathbb{Z}_{p}, b \stackrel{\&}{\leftarrow}\{0,1\}, r_{1}=x^{2}$, and $r_{0} \stackrel{\&}{\leftarrow} \mathbb{Z}_{p}$ are secret choices, output $b$. Our result is formalized in Theorem 2 It is worth mentioning that in contrast to computational problems (like $\mathrm{Co}-\mathrm{DH}$ ) for decisional problems usually multiple reduction steps are required. In the proof we apply the idea of DDH-steps [12] to the bilinear setting and introduce the new concept of SqDDH-steps. Since the $\mathrm{DDH}$ assumption reduces to the SqDDH assumption [38] the hardness of BDDH can be formulated with respect to SqDDH only (Corollary 1 ).

Theorem 2. Suppose there exists a semi-generic group algorithm $\mathcal{A}$ solving BDDH over a Type 1 setting in time $t$ with advantage $\epsilon$. Then there exists an algorithm $\mathcal{B}_{\mathrm{SqDDH}}$ solving $\mathrm{SqDDH}$ over $\mathbb{G}_{3}$ in time $t_{\mathrm{SqDDH}} \approx t$ with advantage $\epsilon_{\mathrm{SqDDH}}$ and an algorithm 
Table 1. Transforming a semi-generic oracle for real BDDH into one for random BDDH using SqDDH and DDH steps

\begin{tabular}{|c|c|c|c|c|c|c|c|c|c|c|}
\hline & $G_{1}$ & $G_{2}$ & $G_{3}$ & $G_{4}$ & $\begin{array}{l}\text { Game } \\
G_{5}\end{array}$ & $G_{6}$ & $G_{7}$ & $G_{8}$ & $G_{9}$ & $G_{10}$ \\
\hline$g_{3}^{r_{b}}$ & $g_{3}^{x_{1} x_{2} x_{3}}$ & $g_{3}^{x_{1} x_{2} x_{3}}$ & $g_{3}^{x_{1} x_{2} x_{3}}$ & $g_{3}^{x_{1} x_{2} x_{3}}$ & $g_{3}^{\mathbf{x} 7^{x_{3}}}$ & $g_{3}^{\mathbf{x} 8}$ & $g_{3}^{x_{8}}$ & $g_{3}^{x_{8}}$ & $g_{3}^{x_{8}}$ & $g_{3}^{x_{8}}$ \\
\hline$e\left(g_{1}, g_{1}\right)$ & $g_{3}$ & $g_{3}$ & $g_{3}$ & $g_{3}$ & $g_{3}$ & $g_{3}$ & $g_{3}$ & $g_{3}$ & $g_{3}$ & $g_{3}$ \\
\hline$e\left(g_{1}, g_{1}^{x_{1}}\right)$ & $g_{3}^{x_{1}}$ & $g_{3}^{x_{1}}$ & $g_{3}^{x_{1}}$ & $g_{3}^{x_{1}}$ & $g_{3}^{x_{1}}$ & $g_{3}^{x_{1}}$ & $g_{3}^{x_{1}}$ & $g_{3}^{x_{1}}$ & $g_{3}^{x_{1}}$ & $g_{3}^{x_{1}}$ \\
\hline$e\left(g_{1}, g_{1}^{x_{2}}\right)$ & $g_{3}^{x_{2}}$ & $g_{3}^{x_{2}}$ & $g_{3}^{x_{2}}$ & $g_{3}^{x_{2}}$ & $g_{3}^{x_{2}}$ & $g_{3}^{x_{2}}$ & $g_{3}^{x_{2}}$ & $g_{3}^{x_{2}}$ & $g_{3}^{x_{2}}$ & $g_{3}^{x_{2}}$ \\
\hline$e\left(g_{1}, g_{1}^{x_{3}}\right)$ & $g_{3}^{x_{3}}$ & $g_{3}^{x_{3}}$ & $g_{3}^{x_{3}}$ & $g_{3}^{x_{3}}$ & $g_{3}^{x_{3}}$ & $g_{3}^{x_{3}}$ & $g_{3}^{x_{3}}$ & $g_{3}^{x_{3}}$ & $g_{3}^{x_{3}}$ & $g_{3}^{x_{3}}$ \\
\hline$e\left(g_{1}^{x_{1}}, g_{1}^{x_{1}}\right)$ & $g_{3}^{x_{1}^{2}}$ & $g_{3}^{\mathbf{x}_{4}}$ & $g_{3}^{x_{4}}$ & $g_{3}^{x_{4}}$ & $g_{3}^{x_{4}}$ & $g_{3}^{x_{4}}$ & $g_{3}^{x_{4}}$ & $g_{3}^{x_{4}}$ & $g_{3}^{x_{4}}$ & $g_{3}^{\mathbf{x}_{1}^{2}}$ \\
\hline$e\left(g_{1}^{x_{2}}, g_{1}^{x_{2}}\right)$ & $g_{3}^{x_{2}^{2}}$ & $g_{3}^{x_{2}^{2}}$ & $g_{3}^{\mathbf{x}_{5}}$ & $g_{3}^{x_{5}}$ & $g_{3}^{x_{5}}$ & $g_{3}^{x_{5}}$ & $g_{3}^{x_{5}}$ & $g_{3}^{x_{5}}$ & $g_{3}^{\mathbf{x}_{2}^{2}}$ & $g_{3}^{x_{2}^{2}}$ \\
\hline$e\left(g_{1}^{x_{3}}, g_{1}^{x_{3}}\right)$ & $g_{3}^{x_{3}^{2}}$ & $g_{3}^{x_{3}^{2}}$ & $g_{3}^{x_{3}^{2}}$ & $g_{3}^{\mathbf{x}_{6}}$ & $g_{3}^{x_{6}}$ & $g_{3}^{x_{6}}$ & $g_{3}^{x_{6}}$ & $g_{3}^{\mathbf{x}_{3}^{2}}$ & $g_{3}^{x_{3}^{2}}$ & $g_{3}^{x_{3}^{2}}$ \\
\hline$e\left(g_{1}^{x_{1}}, g_{1}^{x_{2}}\right)$ & $g_{3}^{x_{1} x_{2}}$ & $g_{3}^{x_{1} x_{2}}$ & $g_{3}^{x_{1} x_{2}}$ & $g_{3}^{x_{1} x_{2}}$ & $g_{3}^{\mathbf{x}_{7}}$ & $g_{3}^{x_{7}}$ & $g_{3}^{\mathbf{x}_{1} \mathbf{x}_{2}}$ & $g_{3}^{x_{1} x_{2}}$ & $g_{3}^{x_{1} x_{2}}$ & $g_{3}^{x_{1} x_{2}}$ \\
\hline$e\left(g_{1}^{x_{1}}, g_{1}^{x_{3}}\right)$ & $g_{3}^{x_{1} x_{3}}$ & $g_{3}^{x_{1} x_{3}}$ & $g_{3}^{x_{1} x_{3}}$ & $g_{3}^{x_{1} x_{3}}$ & $g_{3}^{x_{1} x_{3}}$ & $g_{3}^{x_{1} x_{3}}$ & $g_{3}^{x_{1} x_{3}}$ & $g_{3}^{x_{1} x_{3}}$ & $g_{3}^{x_{1} x_{3}}$ & $g_{3}^{x_{1} x_{3}}$ \\
\hline$e\left(g_{1}^{x_{2}}, g_{1}^{x_{3}}\right)$ & $g_{3}^{x_{2} x_{3}}$ & $g_{3}^{x_{2} x_{3}}$ & $g_{3}^{x_{2} x_{3}}$ & $g_{3}^{x_{2} x_{3}}$ & $g_{3}^{x_{2} x_{3}}$ & $g_{3}^{x_{2} x_{3}}$ & $g_{3}^{x_{2} x_{3}}$ & $g_{3}^{x_{2} x_{3}}$ & $g_{3}^{x_{2} x_{3}}$ & $g_{3}^{x_{2} x_{3}}$ \\
\hline & & SqDDH & SqDDH & $\mathrm{SqDDH}$ & $\begin{array}{c}\text { DDH } \\
\text { Justificati }\end{array}$ & $\begin{array}{l}\text { DDH } \\
\text { on }\end{array}$ & DDH & SqDD & תחט & qDDH \\
\hline
\end{tabular}

$\mathcal{B}_{\mathrm{DDH}}$ solving $\mathrm{DDH}$ over $\mathbb{G}_{3}$ in time $t_{\mathrm{DDH}} \approx t$ with advantage $\epsilon_{\mathrm{DDH}}$ such that $\epsilon \leq$ $3 \epsilon_{\mathrm{DDH}}+6 \epsilon_{\mathrm{SqDDH}}$.

Corollary 1. If SqDDH is $(\epsilon, t)$-hard over $\mathbb{G}_{3}$, then BDDH is $(9 \epsilon, t)$-hard for semigeneric algorithms.

Proof (Theorem 2). In the following we show that a for a semi-generic algorithm a "real" BDDH tuple $\left(g_{1}, g_{1}^{x_{1}}, g_{1}^{x_{2}}, g_{1}^{x_{3}}, g_{3}^{r_{1}}=g_{3}^{x_{1} x_{2} x_{3}}\right)$ is computationally indistinguishable from a "random" tuple $\left(g_{1}, g_{1}^{x_{1}}, g_{1}^{x_{2}}, g_{1}^{x_{3}}, g_{3}^{r_{0}}\right)$, unless SqDDH or DDH are easy over $\mathbb{G}_{3}$. We do this by considering a series of games played between a semi-generic algorithm $\mathcal{A}$ and an oracle $\mathcal{O}$. We start with $\mathcal{A}$ given oracle access to a real BDDH tuple. We then gradually transform this tuple as well as the output of the oracle until we end up with a random tuple. One can show that if $\mathcal{A}$ can distinguish two consecutive games $G_{i-1}$ and $G_{i}$ then it can be used to build an algorithm solving SqDDH or DDH.

The games are described by Table 1 Each of the columns labeled with $G_{i}$ specifies the (direct) input over $\mathbb{G}_{3}$ (see Row 1) or the output of BilinearMap in game $G_{i}$ for all possible inputs over $\mathbb{G}_{1}$. Bold-printed parts of a value highlight the actual changes in comparison to the previous game. The entry in the last row of a column $G_{i}$ indicates which assumption (SqDDH or DDH) justifies the indistinguishability of the Games $G_{i-1}$ and $G_{i}$. If a new $x_{j}(j>3)$ appears in a column, this means that this value has been added to the corresponding game and the oracle chooses $x_{j}$ uniformly from $\mathbb{Z}_{p}$.

As one can see from the table, by means of the Games $G_{2}$ to $G_{4}$ we remove all squares $x_{i}^{2}(1 \leq i \leq 3)$ from the output of the pairing oracle. We do this simply by replacing each square with a new value $x_{j}(4 \leq j \leq 6)$. These transformations are called (bilinear) SqDDH steps and are prerequisites for the subsequent DDH steps performed in Games $G_{5}$ to $G_{6}$. During these DDH steps we selectively remove all 
products $x_{i} x_{j}$ that involve variables being part of the challenge. Again, this is done by replacing the products by fresh uniformly chosen values $x_{j}(j \in\{7,8\})$. In Game $G_{6}$ the challenge $g_{3}^{r_{b}}=g_{3}^{x_{8}}$ is finally independent of the input since $x_{8}$ does not appear anywhere else. After that, in Games $G_{7}$ to $G_{10}$ we reverse the changes we did to the input and BilinearMap during $G_{2}$ to $G_{6}$ in reverse order. More, precisely in $G_{6+j}$ we reverse the changes we did in $G_{6-j}$ for $1 \leq j \leq 4$. Finally, in $G_{10}$ we have reversed all changes (except for the one in $G_{6}$ ). This last game corresponds to the situation where $\mathcal{A}$ is given oracle access to a random BDDH tuple. If all intermediate games have been computationally indistinguishable (under the SqDDH and DDH assumption) then certainly also a real BDDH tuple is computationally indistinguishable from a random tuple, with respect to semi-generic algorithms.

For the sake of clarity, let us consider the transition from $G_{1}$ to $G_{2}$ (SqDDH Step) and $G_{4}$ to $G_{5}$ (DDH Step) in some more detail and quantify the involved reductions. The oracle $\mathcal{O}_{G_{1}}$ in Game $G_{1}$ corresponds to the original semi-generic oracle for BDDH providing access to a real BDDH tuple. The oracle in $\mathcal{O}_{G_{2}}$ in $G_{2}$ is equal to $\mathcal{O}_{G_{1}}$ except for the following changes: $\mathcal{O}_{G_{2}}$ additionally chooses $x_{4} \stackrel{\$}{\leftarrow} \mathbb{Z}_{p}$ and uses a slightly modified table for computing pairing outputs as specified in Table 1 Let us assume $\mathcal{A}$ distinguishes the two games in time $t$ with advantage

$$
\epsilon_{1}=\operatorname{Adv}_{\mathcal{A}}^{G_{1}, G_{2}}=\left|\operatorname{Pr}\left[1 \leftarrow \mathcal{A}^{\mathcal{O}_{G_{1}}}\right]-\operatorname{Pr}\left[1 \leftarrow \mathcal{A}^{\mathcal{O}_{G_{2}}}\right]\right|
$$

Then from $\mathcal{A}$ we can build an algorithm $\mathcal{B}$ for SqDDH. Again, we make use of the observation that semi-generic algorithms are blind with respect to $\mathbb{G}_{1}$ and $e$ and set $\mathbb{G}_{1}:=\mathbb{G}_{3}$ and $e: \mathbb{G}_{3} \times \mathbb{G}_{3} \rightarrow \mathbb{G}_{3}$. Now let an instance

$$
g_{3}, g_{3}^{x_{1}}, g_{3}^{r_{b^{\prime}}^{\prime}}= \begin{cases}g_{3}^{x_{1}^{2}}, & b^{\prime}=1 \\ g_{3}^{x_{4}}, & b^{\prime}=0\end{cases}
$$

of the SqDDH problem over $\mathbb{G}_{3}$ be given. $\mathcal{B}$ chooses $x_{2}, x_{3} \stackrel{\$}{\leftarrow} \mathbb{Z}_{p}$. Then it simulates $\mathcal{O}_{G_{1}}$ and $\mathcal{O}_{G_{2}}$ as follows (we indicate below how group elements are computed though $x_{1}, x_{1}^{2}, x_{4}$, and $b^{\prime}$ are unknown to $\left.\mathcal{B}\right)$ :

- The list $\mathcal{E}_{1}$ is initialized with $g_{3}, g_{3}^{x_{1}}, g_{3}^{x_{2}}, g_{3}^{x_{3}}$. Over $\mathbb{G}_{3} \mathcal{A}$ is given $g_{3},\left(g_{3}^{x_{1}}\right)^{x_{2} x_{3}}$.

- For simulating BilinearMap, we use the fact that we only need to know the pairing output for all possible initial inputs. These elements can be computed as described by the following table:

\begin{tabular}{c|cccccccccc}
$a$ & $g_{3}$ & $g_{3}$ & $g_{3}$ & $g_{3}$ & $g_{3}^{x_{1}}$ & $g_{3}^{x_{2}}$ & $g_{3}^{x_{3}}$ & $g_{3}^{x_{1}}$ & $g_{3}^{x_{1}}$ & $g_{3}^{x_{2}}$ \\
\hline$b$ & $g_{3}$ & $g_{3}^{x_{1}}$ & $g_{3}^{x_{2}}$ & $g_{3}^{x_{3}}$ & $g_{3}^{x_{1}}$ & $g_{3}^{x_{2}}$ & $g_{3}^{x_{3}}$ & $g_{3}^{x_{2}}$ & $g_{3}^{x_{3}}$ & $g_{3}^{x_{3}}$ \\
\hline$e(a, b)$ & $g_{3}$ & $g_{3}^{x_{1}}$ & $g_{3}^{x_{2}}$ & $g_{3}^{x_{3}}$ & $g_{3}^{r_{b^{\prime}}^{\prime}}$ & $g_{3}^{x_{2}^{2}}$ & $g_{3}^{x_{3}^{2}}$ & $\left(g_{3}^{x_{1}}\right)^{x_{2}}$ & $\left(g_{3}^{x_{1}}\right)^{x_{3}}$ & $g_{3}^{x_{2} x_{3}}$
\end{tabular}

It is easy to see that if $b^{\prime}=1$, algorithm $\mathcal{B}$ exactly simulates $\mathcal{O}_{G_{1}}$ and $\mathcal{O}_{G_{2}}$ otherwise. Thus, by simply forwarding the output of $\mathcal{A}, \mathcal{B}$ solves the SqDDH problem instance with the same advantage $\epsilon_{1}$.

Let us now consider the transition from $G_{4}$ to $G_{5}$. The oracle $\mathcal{O}_{G_{5}}$ in $G_{5}$ coincides with $\mathcal{O}_{G_{4}}$ except for the following changes: $\mathcal{O}_{G_{5}}$ additionally chooses $x_{7} \stackrel{\$}{\leftarrow} \mathbb{Z}_{p}$ and 
uses a modified table for computing pairing outputs as specified in Table 1 Assume $\mathcal{A}$ distinguishes the two games in time $t$ with advantage $\epsilon_{4}=\operatorname{Adv}_{\mathcal{A}}^{G_{4}, G_{5}}$. Then we can use $\mathcal{A}$ to build an algorithm $\mathcal{B}$ for $\mathrm{DDH}$. Given an instance

$$
g_{3}, g_{3}^{x_{1}}, g_{3}^{x_{2}}, g_{3}^{r_{b^{\prime}}^{\prime}}= \begin{cases}g_{3}^{x_{1} x_{2}}, & b^{\prime}=1 \\ g_{3}^{x_{7}}, & b^{\prime}=0\end{cases}
$$

of DDH over $\mathbb{G}_{3}, \mathcal{B}$ chooses $x_{3}, x_{4}, x_{5}, x_{6} \stackrel{\$}{\leftarrow} \mathbb{Z}_{p}$ and simulates $\mathcal{O}_{G_{5}}$ and $\mathcal{O}_{G_{6}}$ :

- The list $\mathcal{E}_{1}$ is initialized with $g_{3}, g_{3}^{x_{1}}, g_{3}^{x_{2}}, g_{3}^{x_{3}}$. Over $\mathbb{G}_{3} \mathcal{A}$ is given $g_{3},\left(g_{3}^{r^{\prime}{ }^{\prime}}\right)^{x_{3}}$.

- For simulating BilinearMap we use the following table of pairing outputs:

\begin{tabular}{c|cccccccccc}
$a$ & $g_{3}$ & $g_{3}$ & $g_{3}$ & $g_{3}$ & $g_{3}^{x_{1}}$ & $g_{3}^{x_{2}}$ & $g_{3}^{x_{3}}$ & $g_{3}^{x_{1}}$ & $g_{3}^{x_{1}}$ & $g_{3}^{x_{2}}$ \\
\hline$b$ & $g_{3}$ & $g_{3}^{x_{1}}$ & $g_{3}^{x_{2}}$ & $g_{3}^{x_{3}}$ & $g_{3}^{x_{1}}$ & $g_{3}^{x_{2}}$ & $g_{3}^{x_{3}}$ & $g_{3}^{x_{2}}$ & $g_{3}^{x_{3}}$ & $g_{3}^{x_{3}}$ \\
\hline$e(a, b)$ & $g_{3}$ & $g_{3}^{x_{1}}$ & $g_{3}^{x_{2}}$ & $g_{3}^{x_{3}}$ & $g_{3}^{x_{4}}$ & $g_{3}^{x_{5}}$ & $g_{3}^{x_{6}}$ & $g_{3}^{r_{b^{\prime}}}$ & $\left(g_{3}^{x_{1}}\right)^{x_{3}}$ & $\left(g_{3}^{x_{2}}\right)^{x_{3}}$
\end{tabular}

If $b^{\prime}=1, \mathcal{B}$ behaves like $\mathcal{O}_{G_{4}}$ whereas it behaves like $\mathcal{O}_{G_{5}}$ if $b^{\prime}=0$. By simply forwarding the output of $\mathcal{A}, \mathcal{B}$ solves the DDH problem instance with advantage $\epsilon_{4}$.

The bound on $\epsilon$ follows now from $\epsilon \leq \sum_{i=1}^{9} \epsilon_{i}$, where $\epsilon_{i}=\operatorname{Adv}_{\mathcal{A}}^{G_{i}, G_{i+1}}$, and setting $\epsilon_{\mathrm{SqDDH}}=\max _{i \in\{1,2,3,7,8,9\}}\left(\epsilon_{i}\right), \epsilon_{\mathrm{DDH}}=\max _{i \in\{4,5,6\}}\left(\epsilon_{i}\right)$.

\section{Analysis of General Problem Classes}

Analyzing general problem classes instead of individual problems is important for at least two reasons: First, it improves our understanding of the properties that need to be satisfied by a problem to be intractable with respect to semi-generic algorithms. Second, master theorems for these classes alleviate the burden of analyzing future problems.

Generalized Pairing-Based Problems. Let a Type 1, 2, or 3 setting according to Definition 1 be given. Furthermore, let $\ell \in \mathbb{N}, d \in\{1,2,3\}$ be positive integers, $\mathbf{I}_{1}, \mathbf{I}_{2}, \mathbf{I}_{3} \subset \mathbb{Z}_{p}\left[X_{1}, \ldots, X_{\ell}\right]$ be finite sets of (publicly known) polynomials (called input polynomials) and $Q \in \mathbb{Z}_{p}\left[X_{1}, \ldots, X_{\ell}\right]$ be a single (publicly known) polynomial (called challenge polynomial). Then we define a $\left(\mathbf{I}_{1}, \mathbf{I}_{2}, \mathbf{I}_{3}, Q\right)-\mathrm{BDH}_{\mathbb{G}_{d}}$ problem as: Given

$$
\left(\left(g_{1}^{R(\mathbf{x})}\right)_{R \in \mathbf{I}_{\mathbf{1}}},\left(g_{2}^{R(\mathbf{x})}\right)_{R \in \mathbf{I}_{\mathbf{2}}},\left(g_{3}^{R(\mathbf{x})}\right)_{R \in \mathbf{I}_{\mathbf{3}}}\right),
$$

where $\mathbf{x} \stackrel{\$}{\leftarrow} \mathbb{Z}_{p}^{\ell}$ are secret random values, output $g_{d}^{Q(\mathbf{x})}$. A decisional variant of such problems can be defined analogously. In the following we always assume that the polynomial 1 is contained in each $\mathbf{I}_{\mathbf{i}}$ which corresponds to the natural assumption that for each group a generator is given.

Informally speaking, a $\left(\mathbf{I}_{\mathbf{1}}, \mathbf{I}_{\mathbf{2}}, \mathbf{I}_{\mathbf{3}}, Q\right)-\mathrm{BDH}_{\mathbb{G}_{d}}$ problem is non-trivial if there is no way to compute $Q$ using only the input polynomials and the operations on them which are implicitly given by the underlying bilinear setting. Let us restrict here to consider the case $d \in\{1,2\}$. Let $\mathbf{I}_{\mathbf{1}}=\left\{R_{1}, \ldots, R_{t}\right\}$ and $\mathbf{I}_{\mathbf{2}}=\left\{S_{1}, \ldots, S_{t^{\prime}}\right\}$. Then using 
Observation 2 (Section2.1), one can see that the output $[c]_{d}$ of a semi-generic algorithm for the considered problem can be written as $g_{d}^{P(\mathbf{x})}$ for some $P$ of the form

$$
P= \begin{cases}\sum_{j=1}^{t} z_{j} R_{j}, & d=1 \\ \sum_{j=1}^{t^{\prime}} z_{j}^{\prime} S_{j}, & d=2 \text { and Type } 3 \text { setting } \\ \sum_{j=1}^{t} z_{j} R_{j}+\sum_{j=1}^{t^{\prime}} z_{j}^{\prime} S_{j}, & d=2 \text { and Type } 2 \text { setting }\end{cases}
$$

We call a $\left(\mathbf{I}_{\mathbf{1}}, \mathbf{I}_{\mathbf{2}}, \mathbf{I}_{\mathbf{3}}, Q\right)-\mathrm{BDH}_{\mathbb{G}_{d}}$ non-trivial if there is no $P$ of the above form such that $g_{d}^{P(\mathbf{x})}=g_{d}^{Q(\mathbf{x})}$ for all $\mathbf{x} \in \mathbb{Z}_{p}^{\ell}$, i.e., if $P \neq Q \in \mathbb{Z}_{p}\left[X_{1}, \ldots, X_{\ell}\right]$. More formal and general definitions can be found in the full version of this paper [20].

Reductions for Generalized Problems. Theorem 3 extends the reduction we have seen for Co-DH in Section 3.1 to the more general class of $\left(\mathbf{I}_{\mathbf{1}}, \mathbf{I}_{\mathbf{2}}, \mathbf{I}_{\mathbf{3}}, Q\right)-\mathrm{BDH}_{\mathbb{G}_{d}}$ problems. The crucial difference and novelty lies in the technique for extracting the wanted discrete logarithm given the output of the semi-generic algorithm.

Theorem 3. Let $d \in\{1,2\}$ and $\left(\mathbf{I}_{\mathbf{1}}, \mathbf{I}_{\mathbf{2}}, \mathbf{I}_{\mathbf{3}}, Q\right)-\mathrm{BDH}_{\mathbb{G}_{d}}$ be a non-trivial problem with challenge and input polynomials in $\mathbb{Z}_{p}\left[X_{1}, \ldots, X_{\ell}\right]$. Let $k=\max _{i}\left(\operatorname{deg}_{X_{i}}\left(\mathbf{I}_{\mathbf{1}} \cup \mathbf{I}_{\mathbf{2}} \cup\right.\right.$ $\left.\left.\mathbf{I}_{3}\right)\right)$. Suppose there is a semi-generic algorithm $\mathcal{A}$ solving $\left(\mathbf{I}_{1}, \mathbf{I}_{\mathbf{2}}, \mathbf{I}_{\mathbf{3}}, Q\right)-\mathrm{BDH}_{\mathbb{G}_{d}}$ in time $t$ with success probability $\epsilon$. Then there is an algorithm $\mathcal{B}$ solving $2 k$-DL in $\mathbb{G}_{3}$ in time $t^{\prime} \approx t+\tilde{O}\left(k^{\prime} \log p\right)$, where $k^{\prime}=\max (k, \operatorname{deg}(Q))$, with probability $\epsilon^{\prime} \geq \frac{\epsilon}{\ell}$.

Proof. Let $k_{1}=2 k$. $\mathcal{B}$ takes as input a $k_{1}$-DL challenge $a_{0}=g_{3}, a_{1}=g_{3}^{x^{1}}, \ldots, a_{k_{1}}=$ $g_{3}^{x^{k_{1}}}$. It then chooses $i^{*} \stackrel{\$}{\leftarrow}\{1, \ldots, \ell\}$ and $x_{1}, \ldots, x_{i^{*}-1}, x_{i^{*}+1}, \ldots, x_{\ell} \stackrel{\$}{\leftarrow} \mathbb{Z}_{p}$. The unknown $x$ is treated as the secret choice $x_{i^{*}}$ in the context of a $\left(\mathbf{I}_{\mathbf{1}}, \mathbf{I}_{\mathbf{2}}, \mathbf{I}_{\mathbf{3}}, Q\right)-\mathrm{BDH}_{\mathbb{G}_{d}}$ instance. We only sketch important points in the simulation of the semi-generic oracle: Each internal list $\mathcal{E}_{j}$ is initialized with the elements $\left(g_{3}^{P(\mathbf{x})}\right)_{P \in \mathbf{I}_{\mathbf{j}}}$ where for a polynomial $P=\sum_{e=\left(e_{1}, \ldots, e_{\ell}\right) \in E} b_{e} X_{1}^{e_{1}} \cdots X_{\ell}^{e_{\ell}}, E \subset \mathbb{Z}_{p}^{\ell}$, the element $g_{3}^{P(\mathbf{x})}$ can be computed as $g_{3}^{P(\mathbf{x})}=\prod_{e} a_{e_{i^{*}}}^{b_{e} \prod_{s \neq i^{*}} x_{s}^{e_{s}}}$ using the given instance of the $k_{1}$-DL problem. This is possible because the degree in $X_{i^{*}}$ of the polynomials in each set $\mathbf{I}_{\mathbf{j}}$ is upper bounded by $k_{1}$. Similarly, the table for simulating BilinearMap can be created since for each entry $g_{3}^{P(\mathbf{x})}$ in this table, $P$ is again of degree at most $k_{1}$ in $X_{i^{*}}$.

Given an $\left(\mathbf{I}_{\mathbf{1}}, \mathbf{I}_{\mathbf{2}}, \mathbf{I}_{\mathbf{3}}, Q\right)-\mathrm{BDH}_{\mathbb{G}_{d}}$ instance, $\mathcal{A}$ eventually outputs an index $[c]_{d}$. Then $c$ can be written as $g_{3}^{P(\mathbf{x})}$ for some known polynomial $P$ as described in Equation 1 Thus, $\mathcal{A}$ wins if $Q(\mathbf{x}) \equiv P(\mathbf{x}) \bmod p$. Since $Z:=Q-P$ is not zero modulo $p$ (the problem is non-trivial) this success event can be split into disjoint events $\mathcal{S}_{1}, \ldots, \mathcal{S}_{\ell}$, where $\mathcal{S}_{j}$ is defined as:

$$
Z\left(X_{1}=x_{1}, \ldots, X_{j-1}=x_{j-1}\right) \not \equiv 0 \text { and } Z\left(X_{1}=x_{1}, \ldots, X_{j}=x_{j}\right) \equiv 0
$$

Denoting the probability of event $\mathcal{S}_{j}$ by $\alpha_{j}$ we obtain $\epsilon=\alpha_{1}+\cdots+\alpha_{\ell}$.

Now assume that event $S_{i^{*}}$ occurs, which happens with probability $\epsilon / \ell$. Consider the polynomial $Z_{i^{*}}=Z\left(X_{1}=x_{1}, \ldots, X_{i^{*}-1}=x_{i^{*}-1}\right) \bmod p \in \mathbb{Z}_{p}\left[X_{i^{*}}, \ldots, X_{\ell}\right]$. This polynomial is of the form $Z_{i^{*}}=\sum_{e=\left(e_{i^{*}}, \ldots, e_{\ell}\right) \in E} b_{e} X_{i^{*}}^{e_{i^{*}}} \cdots X_{\ell}^{e_{\ell}}$, for some $E \subset$ $\mathbb{Z}_{p}^{\ell-i^{*}+1}$, where in at least one monomial the variable $X_{i^{*}}$ appears with a non-zero 
exponent $e_{i^{*}}$. Let $M=b_{e}^{\prime} X_{i^{*}}^{e_{i *}^{\prime}} \cdots X_{\ell}^{e_{\ell}^{\prime}}$ be one of these monomials. Then consider the polynomial $Z_{i^{*}}^{\prime}$ we obtain by summing up all monomials of $Z_{i^{*}}$ containing the submonomial $X_{i^{*}+1}^{e^{\prime *}+1} \cdots X_{\ell}^{e_{\ell}^{\prime}}$ :

$$
Z_{i^{*}}^{\prime}=\sum_{\substack{e=\left(e_{i^{*}}, \ldots, e_{\ell}\right) \in E \\ e_{i^{*}+1}=e_{i^{*}+1}^{\prime}, \ldots, e_{\ell}=e_{\ell}^{\prime}}} b_{e} X_{i^{*}}^{e_{i^{*}}} X_{i^{*}}^{e_{i^{*}}^{\prime}} \cdots X_{\ell}^{e_{\ell}^{\prime}}
$$

Clearly, we have $Z_{i^{*}}^{\prime} \not \equiv 0 \bmod p$ and since $Z_{i^{*}}\left(X_{i^{*}}=x_{i^{*}}\right) \equiv 0 \bmod p$ it also holds that $Z_{i^{*}}^{\prime}\left(X_{i^{*}}=x_{i^{*}}\right) \equiv 0 \bmod p$. Hence, $x_{i^{*}}=x$ is a root of the non-zero uni-variate polynomial

$$
Z_{i^{*}}^{\prime \prime}=\sum_{\substack{e=\left(e_{i *}^{*}, \ldots, e_{\ell}\right) \in E \\ e_{i^{*}+1}=e_{i^{*}+1}^{\prime}, \ldots, e_{\ell}=e_{\ell}^{\prime}}} b_{e} X_{i^{*}}^{e_{i *}}
$$

Note that Algorithm $\mathcal{B}$ can easily construct the polynomial $Z_{i^{*}}^{\prime \prime}$ by picking an arbitrary monomial from $Z_{i^{*}}$ for which $X_{i^{*}}$ appears with non-zero exponent. The coefficients $b_{e}$ can also be easily computed since the coefficients of $Z$ are known and $x_{1}, \ldots, x_{i^{*}-1}$ have been chosen by $\mathcal{B}$. So by applying an efficient standard algorithm for computing roots of polynomials over $\mathbb{Z}_{p}$, such as [36, Algorithm 14.15], $\mathcal{B}$ can find the wanted DL $x_{i^{*}}=x$ by computing all roots of the polynomial $Z_{i^{*}}^{\prime \prime}$. These at most $k^{\prime}=\max (k, \operatorname{deg}(Q))$ different roots can be computed in time $\tilde{O}\left(k^{\prime} \log p\right)$ [36, Corollary 14.16]. Whether a root $x^{\prime}$ equals $x$ can be tested by verifying $g^{x^{\prime}} \stackrel{?}{=} a_{1}$.

We have also been able to find a reduction for a general class of decisional problems which is efficient for virtually all problems of this class considered in practice. Essentially, our reduction from the SqDDH problem over $\mathbb{G}_{3}$ works for all $\left(\mathbf{I}_{\mathbf{1}}, \mathbf{I}_{\mathbf{2}}, \mathbf{I}_{\mathbf{3}}, Q\right)$ $\mathrm{BDDH}_{\mathbb{G}_{3}}$ problems where variables in $\mathbf{I}_{\mathbf{1}} \cup \mathbf{I}_{\mathbf{2}}$ and $\mathbf{I}_{\mathbf{3}} \cup\{Q\}$ appear with at most linear and quadratic exponents, respectively. Our approach for this general reduction differs from the one for BDDH we have seen in Section 3.2 in the following way: The BDDH reduction is direct in the sense that all reduction steps take place directly in the semigeneric model. As an alternative, one could also first "project" BDDH to the group $\mathbb{G}_{3}$ by finding an "appropriate" problem which reduces in a single step to BDDH (with respect to semi-generic algorithms) and then apply all DDH and SqDDH reduction steps to this problem in the standard model. We follow this latter approach in our proof for general bilinear decisional problems since it has the advantage that we can resort to Bresson et al.'s results for generalized DDH problems [12] in the standard model. However, this is not straightforward. Since their results are quite restricted we need to enhance them to more general problem classes. For more details on our result for bilinear decisional problems we refer to the full version [20].

\section{Analyzing Cryptosystems in the Semi-Generic Model}

Besides for studying cryptographic hardness assumptions, it would also be interesting to use the SGGM as a tool to analyze the security of practical pairing-based cryptosystems. Similar analyzes have been made in the classical GGM [35]13]. In this section 
we consider the Boneh-Lynn-Shacham (BLS) signature scheme [9|10] in the SGGM. It turns out that it is possible to prove security of this scheme under the semi-generic groups heuristic, by requiring concrete (but non-standard) properties of the hash function.

The BLS signature scheme over a Type 1 bilinear setting is defined as follows. Let $H_{1}$ be a hash function $H_{1}:\{0,1\}^{\ell} \rightarrow \mathbb{G}_{1}$.

- Gen samples a random generator $g$ of $\mathbb{G}_{1}, s \stackrel{\$}{\leftarrow} \mathbb{Z}_{p}$, and sets $p k=\left(g, g^{s}\right)$, sk $=s$.

- Sign $(s k, m)$ computes $H_{1}(m)$ and returns $\sigma=H_{1}(m)^{s}$.

- $\operatorname{Verify}(p k, m, \sigma)$ returns 1 , if $e\left(H_{1}(m), p k\right)=e(\sigma, g)$, and 0 otherwise.

Let us now describe the EUF-CMA security experiment for the BLS signature scheme in the SGGM. Here we are facing a technical problem: the BLS scheme utilizes a hash function $H_{1}:\{0,1\}^{\ell} \rightarrow \mathbb{G}_{1}$, that is, the output of this map is a group element in some given representation. However, in the SGGM we want to consider algorithms which are independent of a particular representation of elements of $\mathbb{G}_{1}$. Since in our model elements of $\mathbb{G}_{1}$ are given as list indices, we have no representation of group elements that we could use as the range of the hash function.

One possible solution would be to fall back on the formalization of a generic group by Shoup [34]. In this model, group elements are represented by unique random bit strings. Thus, we could use a hash function that maps to bit strings of appropriate size. However, the fact that group elements are encoded as random strings has been subject to much criticism [16 2615]. For instance, the Shoup model can be misused to implement a random oracle, which is of no avail since we want to avoid random oracles in our security proof. Therefore we follow a different approach. We implement $H_{1}$ as a generic group hash function.

Definition 2. A group hash function is a pair of algorithms $\mathbf{H}=(\mathrm{GHGen}, \mathrm{GHEval})$.

- GHGen takes as input a generator $g$ of $\mathbb{G}_{1}$, and returns $A=\left(a_{1}, \ldots, a_{\delta}\right) \in \mathbb{G}_{1}^{\delta}$. Vector A specifies a function $H_{1}:\{0,1\}^{\ell} \rightarrow \mathbb{G}_{1}$.

- Algorithm GHEval takes as input a vector $A \in \mathbb{G}_{1}^{\delta}$ and a string $m \in\{0,1\}^{\ell}$, and returns $H_{1}(m) \in \mathbb{G}_{1}$.

We say that a group hash function is generic, if GHGen and GHEval perform only group operations on elements of $A$.

Examples of generic group hash functions are the hash function used in Water's IBE scheme [37] and the programmable hash functions of Hofheinz and Kiltz [18].

Generic group hash functions have the useful property that there exist "trapdoor" set-up and evaluation algorithms (TrapGen, TrapEval) with the following properties.

- TrapGen takes as input a generator $g \in \mathbb{G}_{1}$. It returns a vector $A \in \mathbb{G}_{1}^{\delta}$, distributed identically to the output of GHGen for all $g$, and some trapdoor information $t d$.

- Algorithm TrapEval takes as input a vector $A \in \mathbb{G}_{1}^{\delta}$ and a string $m \in\{0,1\}^{\ell}$, and returns $h$ such that $g^{h}=H_{1}(m)$.

For the security proof we need to demand a strong form of collision resistance. 
Definition 3. A group hash function is $(\epsilon, t, q)$-algebraic collision resistant, if

$$
\operatorname{Pr}\left[\mathcal{A}(A)=\left(m_{0}, \ldots, m_{q}, i_{0}, \ldots, i_{q}\right): H_{1}\left(m_{0}\right)=g^{i_{0}} \prod_{j=1}^{q}\left(H_{1}\left(m_{j}\right)\right)^{i_{j}}\right] \leq \epsilon
$$

for all algorithms $\mathcal{A}$ running in time $t$.

By employing techniques from [18] it is possible to construct hash functions satisfying this property under weak assumptions, like the hardness of computing discrete logarithms in $\mathbb{G}_{1}$, for any constant $q$. A major drawback is, however, that for these constructions the size $\delta$ of vector $A$ grows at least linearly with $q$. We leave it as an open problem to study whether there exists a (possibly probabilistic) trapdoor group hash function such that $\delta$ is constant and $q=q(\kappa)$ is a polynomial.

We formalize the EUF-CMA experiment in the SGGM as follows. At the beginning of the game, the challenger samples a random generator $g$ and a secret key $x$. Then it runs $\left(a_{1}, \ldots, a_{\delta}\right) \stackrel{\$}{\leftarrow} \mathrm{GHGen}(g)$, sets $I_{1}:=\left(g, g^{x}, a_{1}, \ldots, a_{\delta}\right)$, and implements a semigeneric oracle with input $I_{1}$ as described in Section 2. This provides the adversary with the public key, and the ability to perform group operations on elements of $\mathbb{G}_{1}$.

When the adversary queries a signature for some chosen message $m_{i}$, the challenger computes $H\left(m_{i}\right)^{x}$ and appends it to the list $\mathcal{E}_{1}$.

We say that the adversary wins the game, if it outputs a message $m$ and index $[s]_{1}$ such that $s=H(m)^{x}$, that is, the adversary has computed a valid signature for $m$. We say that a semi-generic adversary $\mathcal{A}(\epsilon, t)$-breaks the EUF-CMA security of a signature scheme if $\mathcal{A}$ runs in time $t$ and $\operatorname{Pr}[\mathcal{A}$ wins $] \geq \epsilon$.

Theorem 4. Suppose there exists an adversary $\mathcal{A}(\epsilon, t)$-breaking the EUF-CMA security of the BLS signature scheme in the semi-generic model by making $q$ chosenmessage signature queries. Then there exists an algorithm $\mathcal{B}_{\mathrm{coll}}\left(\epsilon_{\mathrm{dl}}, t_{\mathrm{dl}}, q\right)$-breaking the algebraic collision resistance of $H_{1}$ and an algorithm $\mathcal{B}_{\mathrm{dl}}\left(\epsilon_{\mathrm{dl}}, t_{\mathrm{dl}}\right)$-solving the discrete logarithm problem in $\mathbb{G}_{1}$, such that $t \approx t_{\mathrm{coll}} \approx t_{\mathrm{dl}}$ and $\epsilon \leq \epsilon_{\mathrm{coll}}+\epsilon_{\mathrm{dl} \text {. }}$.

Proof. Suppose there exists an adversary $\mathcal{A}$ that outputs a message $m$ and an index $[s]_{1}$ such that $s=H(m)^{x}$. In the SGGM, an adversary has to compute a group element of $\mathbb{G}_{1}$ by applying a sequence of group operations to the initial values $\left(g, g^{x}, a_{1}, \ldots, a_{\delta}\right)$ stored in $\mathcal{E}_{1}$ and to group elements added to the list by the challenger oracle in response to chosen-message signature queries. Thus, when $\mathcal{A}$ outputs $\left(m,[s]_{1}\right)$ such that $s=$ $H(m)^{x}$, then the oracle obtains an equation

$$
H(m)^{x}=g^{\alpha_{1}} \cdot\left(g^{x}\right)^{\alpha_{2}} \cdot \prod_{i=1}^{\delta} a_{i}^{\beta_{i}} \cdot \prod_{i=1}^{q}\left(H\left(m_{i}\right)^{x}\right)^{\gamma_{i}},
$$

or equivalently $x \cdot\left(\log _{g} H(m)-\sum_{i=1}^{q} \gamma_{i} \log _{g} H\left(m_{i}\right)-\alpha_{2}\right)=\alpha_{1}+\sum_{i=1}^{\delta} \beta_{i} \log _{g} a_{i}$, for integers $\alpha_{i}, \beta_{i}, \gamma_{i}$ known to the oracle. We consider two types of forgers:

1. A Type-A forger performs a sequence of operations such that

$$
\log _{g} H(m)-\sum_{i=1}^{q} \gamma_{i} \log _{g} H\left(m_{i}\right)-\alpha_{2} \equiv 0 \bmod p .
$$


2. A Type-B forger performs a sequence of operations such that

$$
\log _{g} H(m)-\sum_{i=1}^{q} \gamma_{i} \log _{g} H\left(m_{i}\right)-\alpha_{2} \not \equiv 0 \bmod p .
$$

Lemma 1. Suppose there exists a Type-A forger $\mathcal{A}(\epsilon, t)$-breaking the EUF-CMA security of the BLS signature scheme by making at most $q$ chosen-message queries. Then there exists an algorithm $\mathcal{B}_{\mathrm{coll}}\left(\epsilon_{\mathrm{dl}}, t_{\mathrm{dl}}, q\right)$-breaking the algebraic collision resistance of (GHGen, GHEval) in time $t^{\prime} \approx t$ with success probability $\epsilon_{\mathrm{coll}} \geq \epsilon$.

ProOF. Algorithm $\mathcal{B}_{\text {coll }}$ receives as input a vector $A^{\prime}=\left(g^{\prime}, a_{1}^{\prime}, \ldots, a_{\delta}^{\prime}\right)$. It proceeds exactly like the semi-generic EUF-CMA challenger, except that it sets $g:=g^{\prime}$ and $a_{i}:=a_{i}^{\prime}$ instead of sampling $g$ at random and generating $A$ by running $\operatorname{GHGen}(g)$. Thus, in particular $\mathcal{B}_{\text {coll }}$ chooses the secret key $x \stackrel{\$}{\leftarrow} \mathbb{Z}_{p}$ and thus is able to simulate the original challenger perfectly.

When $\mathcal{A}$ outputs $\left(m,[s]_{1}\right)$ such that $s=H(m)^{x}$, then $\mathcal{B}_{\text {coll }}$ computes and returns integers $\left(\alpha_{2}, \gamma_{1}, \ldots, \gamma_{q}\right)$ as in Equation 4 Observe that if Equation 4 is satisfied, then we have $H(m)=g^{\alpha_{2}} \cdot \prod_{i=1}^{q} H\left(m_{i}\right)^{\gamma_{i}}$.

Lemma 2. Suppose there exists a Type-B forger $\mathcal{A}(\epsilon, t)$-breaking the EUF-CMA security of the $B L S$ signature scheme. Then there exists an algorithm $\mathcal{B}_{\mathrm{dl}}$ solving the discrete logarithm problem in $\mathbb{G}_{1}$ in time $t_{\mathrm{dl}} \approx t$ with success probability $\epsilon_{\mathrm{dl}} \geq \epsilon$.

PROOF. Algorithm $\mathcal{B}_{\mathrm{dl}}$ receives as input a tuple $\left(g^{\prime}, y\right)$. It sets $g:=g^{\prime}, g^{x}:=y$, and runs $(A, t d) \stackrel{\$}{\leftarrow} \operatorname{TrapGen}(g)$ to generate the public parameters of the hash function. Recall that $A$ is distributed identically to some $A^{\prime}$ generated by GHGen. It sets $I_{1}:=$ $\left(g, g^{x}, a_{1}, \ldots, a_{\delta}\right)$, and implements a semi-generic oracle with initial list state $I_{1}$.

Since $\mathcal{B}_{\mathrm{dl}}$ does not know the secret-key exponent $x$, it answers chosen-message signature queries of $\mathcal{A}$ differently. $\mathcal{B}_{\mathrm{dl}}$ makes use of the trapdoor information $t d$ generated along with $A$. Whenever $\mathcal{A}$ submits a chosen-message $m_{i}, \mathcal{B}_{\mathrm{dl}}$ computes $h_{i}=$ $\operatorname{TrapEval}\left(m_{i}\right)$ and appends $y^{h_{i}}$ to $\mathcal{E}_{1}$. Note that $y^{h_{i}}=g^{x \log _{g} H\left(m_{i}\right)}=H\left(m_{i}\right)^{x}$, thus this is a valid signature.

When $\mathcal{A}$ outputs $\left(m,[s]_{1}\right)$ such that $s=H(m)^{x}$, then $\mathcal{B}_{\mathrm{dl}}$ computes integers $\left(\alpha_{i}\right.$, $\left.\beta_{i}, \gamma_{i}\right)$ as in Equation 3, and returns

$$
x=\log _{g^{\prime}} y=\frac{\alpha_{1}+\sum_{i=1}^{\delta} \beta_{i} \log _{g} a_{i}}{\log _{g} H(m)-\sum_{i=1}^{q} \gamma_{i} \log _{g} H\left(m_{i}\right)-\alpha_{2}} \bmod p,
$$

which is possible since $\log _{g} H(m)-\sum_{i=1}^{q} \gamma_{i} \log _{g} H\left(m_{i}\right)-\alpha_{2} \neq 0 \bmod p$.

Acknowledgements. We would like to thank Dennis Hofheinz, Jesper Buus Nielsen, and Dominique Unruh for valuable discussions and the anonymous reviewers of Asiacrypt 2010 for their detailed and helpful comments. 


\section{References}

1. Aggarwal, D., Maurer, U.: Breaking RSA generically is equivalent to factoring. In: Joux, A. (ed.) EUROCRYPT 2009. LNCS, vol. 5479, pp. 36-53. Springer, Heidelberg (2010)

2. Bellare, M., Rogaway, P.: Random oracles are practical: A paradigm for designing efficient protocols. In: ACM Conference on Computer and Communications Security, pp. 62-73 (1993)

3. Boneh, D.: Number-theoretic assumptions. Invited Talk at TCC's Special Session on Assumptions for Cryptography (2007)

4. Boneh, D., Boyen, X.: Efficient selective-id secure identity-based encryption without random oracles. In: Cachin, C., Camenisch, J. (eds.) EUROCRYPT 2004. LNCS, vol. 3027, pp. 223 238. Springer, Heidelberg (2004)

5. Boneh, D., Boyen, X., Goh, E.: Hierarchical identity based encryption with constant size ciphertext (full paper). Cryptology ePrint Archive, Report 2005/015 (2005), http://eprint.iacr.org/

6. Boneh, D., Boyen, X., Shacham, H.: Short group signatures. In: Franklin, M.K. (ed.) CRYPTO 2004. LNCS, vol. 3152, pp. 41-55. Springer, Heidelberg (2004)

7. Boneh, D., Franklin, M.K.: Identity-based encryption from the Weil pairing. In: Kilian, J. (ed.) CRYPTO 2001. LNCS, vol. 2139, pp. 213-229. Springer, Heidelberg (2001)

8. Boneh, D., Gentry, C., Lynn, B., Shacham, H.: Aggregate and verifiably encrypted signatures from bilinear maps. In: Biham, E. (ed.) EUROCRYPT 2003. LNCS, vol. 2656, pp. 416-432. Springer, Heidelberg (2003)

9. Boneh, D., Lynn, B., Shacham, H.: Short signatures from the Weil pairing. In: Boyd, C. (ed.) ASIACRYPT 2001. LNCS, vol. 2248, pp. 514-532. Springer, Heidelberg (2001)

10. Boneh, D., Lynn, B., Shacham, H.: Short signatures from the Weil pairing. J. Cryptology 17(4), 297-319 (2004)

11. Boyen, X.: The Uber-Assumption family. In: Galbraith, S.D., Paterson, K.G. (eds.) Pairing 2008. LNCS, vol. 5209, pp. 39-56. Springer, Heidelberg (2008)

12. Bresson, E., Lakhnech, Y., Mazaré, L., Warinschi, B.: A generalization of DDH with applications to protocol analysis and computational soundness. In: Menezes, A. (ed.) CRYPTO 2007. LNCS, vol. 4622, pp. 482-499. Springer, Heidelberg (2007)

13. Brown, D.R.L.: Generic groups, collision resistance, and ECDSA. Des. Codes Cryptography 35(1), 119-152 (2005)

14. Cheon, J.: Security analysis of the Strong Diffie-Hellman problem. In: Vaudenay, S. (ed.) EUROCRYPT 2006. LNCS, vol. 4004, pp. 1-11. Springer, Heidelberg (2006)

15. Dent, A.W.: Adapting the weaknesses of the random oracle model to the generic group model. In: Zheng, Y. (ed.) ASIACRYPT 2002. LNCS, vol. 2501, pp. 100-109. Springer, Heidelberg (2002)

16. Fischlin, M.: A note on security proofs in the generic model. In: Okamoto, T. (ed.) ASIACRYPT 2000. LNCS, vol. 1976, pp. 458-469. Springer, Heidelberg (2000)

17. Galbraith, S.D., Paterson, K.G., Smart, N.P.: Pairings for cryptographers. Discrete Applied Mathematics 156(16), 3113-3121 (2008)

18. Hofheinz, D., Kiltz, E.: Programmable hash functions and their applications. In: Wagner, D. (ed.) CRYPTO 2008. LNCS, vol. 5157, pp. 21-38. Springer, Heidelberg (2008)

19. Hohenberger, S.: The cryptographic impact of groups with infeasible inversion. Master's thesis, Massachusetts Institute of Technology (2003)

20. Jager, T., Rupp, A.: The semi-generic group model and applications to pairing-based cryptography (full paper) (2010), http: / / www .nds.rub.de/chair/publications /

21. Jager, T., Schwenk, J.: On the analysis of cryptographic assumptions in the generic ring model. In: Matsui, M. (ed.) ASIACRYPT 2009. LNCS, vol. 5912, pp. 399-416. Springer, Heidelberg (2009) 
22. Jao, D., Yoshida, K.: Boneh-Boyen signatures and the Strong Diffie-Hellman problem. Cryptology ePrint Archive, Report 2009/221 (2009), http: / /eprint.iacr.org/

23. Joux, A.: A one round protocol for tripartite Diffie-Hellman. In: Bosma, W. (ed.) ANTS 2000. LNCS, vol. 1838, pp. 385-394. Springer, Heidelberg (2000)

24. Joux, A.: A one round protocol for tripartite Diffie-Hellman. J. Cryptology 17(4), 263-276 (2004)

25. Katz, J., Sahai, A., Waters, B.: Predicate encryption supporting disjunctions, polynomial equations, and inner products. In: Smart, N.P. (ed.) EUROCRYPT 2008. LNCS, vol. 4965 , pp. 146-162. Springer, Heidelberg (2008)

26. Koblitz, N., Menezes, A.: Another look at generic groups. Advances in Mathematics of Communications 1, 13-28 (2007)

27. Leander, G., Rupp, A.: On the equivalence of RSA and factoring regarding generic ring algorithms. In: Lai, X., Chen, K. (eds.) ASIACRYPT 2006. LNCS, vol. 4284, pp. 241-251. Springer, Heidelberg (2006)

28. Maurer, U.: Abstract models of computation in cryptography. In: Smart, N.P. (ed.) Cryptography and Coding 2005. LNCS, vol. 3796, pp. 1-12. Springer, Heidelberg (2005)

29. Menezes, A., Okamoto, T., Vanstone, S.: Reducing elliptic curve logarithms to logarithms in a finite field. IEEE Transactions on Information Theory 39(5), 1639-1646 (1993)

30. Pohlig, S.C., Hellman, M.E.: An improved algorithm for computing logarithms over $G F(p)$ and its cryptographic significance. IEEE Transactions on Information Theory 24, 106-110 (1978)

31. Pollard, J.M.: Monte Carlo methods for index computation mod $p$. Mathematics of Computation 32, 918-924 (1978)

32. Rivest, R.L.: On the notion of pseudo-free groups. In: Naor, M. (ed.) TCC 2004. LNCS, vol. 2951, pp. 505-521. Springer, Heidelberg (2004)

33. Rupp, A., Leander, G., Bangerter, E., Dent, A.W., Sadeghi, A.: Sufficient conditions for intractability over black-box groups: Generic lower bounds for generalized DL and DH problems. In: Pieprzyk, J. (ed.) ASIACRYPT 2008. LNCS, vol. 5350, pp. 489-505. Springer, Heidelberg (2008)

34. Shoup, V.: Lower bounds for discrete logarithms and related problems. In: Fumy, W. (ed.) EUROCRYPT 1997. LNCS, vol. 1233, pp. 256-266. Springer, Heidelberg (1997)

35. Smart, N.P.: The exact security of ECIES in the generic group model. In: Honary, B. (ed.) Cryptography and Coding 2001. LNCS, vol. 2260, pp. 73-84. Springer, Heidelberg (2001)

36. von zur Gathen, J., Gerhard, J.: Modern Computer Algebra, 2nd edn. Cambridge University Press, Cambridge (2003)

37. Waters, B.: Efficient identity-based encryption without random oracles. In: Cramer, R. (ed.) EUROCRYPT 2005. LNCS, vol. 3494, pp. 114-127. Springer, Heidelberg (2005)

38. Wolf, S.: Information-theoretically and computationally secure key agreement in cryptography. PhD thesis, ETH Zurich, ETH dissertation No. 13138 (1999) 